


\section{Editor of The ANZIAM Journal}

C. E. M. PEARCE, Department of Applied Mathematics, The University of Adelaide, Australia, 5005; cpearce@maths.adelaide.edu.au .

\section{Associate Editors}

A. P. BASSOM
Department of Mathematics
University of Exeter
North Park Road
Exeter, Devon EX4QE
United Kingdom
AP.Bassom@ex.ac.uk
A. J. BRACKEN
Department of Mathematics
University of Queensland
St. Lucia QLD 4072
ajb@ maths.uq.edu.au
B. D. CRAVEN
Department of Mathematics
University of Melbourne
VIC 3010
craven@ms.unimelb.edu.au

A. C. EbERHARD

Department of Mathematics

Royal Melbourne Institute of

Technology

GPO Box 2476V

Melboume VIC 3001

andrew.eberhard@ems.rmit.edu.au

L. K. FORBES

School of Mathematics

and Physics

The University of Tasmania

Hobart TAS 7001

Larry.Forbes@utas.edu.au

J. M. HILL

Department of Mathematics

University of Wollongong

PO Box 1144

Wollongong NSW 2500

j.hill@uow.edu.au

G. C. HockING

Department of Mathematics

and Statistics

Murdoch University

Murdoch WA 6150

hocking@maths.uwa.edu.au

\section{A. Hurst}

Department of Physics and

Mathematical Physics

The University of Adelaide

Australia 5005

ahurst@physics.adelaide.edu.au

P. E. KLOEDEN

Fachbereich Mathematik

Johann Wolfgang Goethe

Universität

D-60054 Frankfurt am Main

Germany

kloeden@math.uni-frankfurt.de

R. MCKIBBIN

Department of Mathematics

Massey University

Private Bag 11222

Palmerston North

New Zealand

R.McKibbin@massey.ac.nz

W. MCLEAN

Department of Applied

Mathematics

University of New South Wales

Sydney NSW 2052

W.Mclean@unsw.edu.au

\section{P. K. POLleTt}

Department of Mathematics

University of Queensland

QLD 4072

pkp@maths.uq.edu.au

D. RALPH

The Judge Institute of

Management Studies

Cambridge University

Trumpington St

Cambridge CB2 1AG

England

dr241@cam.ac.uk
H. M. SRIVASTAVA

Department of Mathematics and Statistics

University of Victoria

Victoria B.C. V8W 3P4

Canada

hmsri@uvvm.uvic.ca

K. L. TEO

Department of Applied

Mathematics

The Hong Kong Polytechnic

University

Hung Hom, Kowloon

Hong Kong

mateokl@polyu.edu.hk

A. TORDEsillas

Department of Mathematics

University of Melbourne

VIC 3010

atordesi@mundoe.maths.mu.oz.au

J.-M. VANDEN-BROECK

School of Mathematics

University of East Anglia

Norwich NR4 7TJ

England

J.Vanden-Broeck@uea.ac.uk

G. C. WAKE

Department of Mathematics

and Statistics

University of Canterbury

Private Bag 4800

Christchurch

New Zealand

g.wake@math.canterbury.ac.nz

G. J. WEIR

Applied Mathematics Centre

Industrial Research Lid

PO Box 31310

Lower Hutt

New Zealand

g.weir@irl.cri.nz

\section{Executive Editor}

E. HUNT, Department of Applied Mathematics, The University of Adelaide, Australia, 5005;

ehunt@maths.adelaide.edu.au .

\section{Assistant Editors}

\section{J. P. DENIER and Y. STOKES}

Department of Applied Mathematics

The University of Adelaide, Australia, 5005.

Cover design by HILARY BOOTH.
A. GILL

Land Operations Division, DSTO

PO Box 1500, Salisbury, SA 5108. 


\title{
THE ANZIAM JOURNAL
}

\author{
Volume 42 Part 4 \\ APRIL 2001
}

Towards numerically estimating Hausdorff dimensions

David E. Stewart

Equilibrium with fixed budgets and superlinear connections

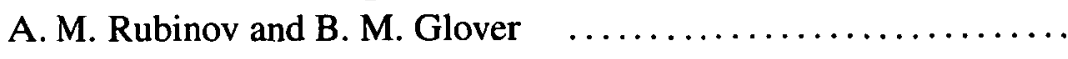

Early quasi-steady electro-magnetic fields about conducting surfaces

Graham J. Weir

Bilateral estimates of the critical Mach number for some classes of carrying wing profiles

F. G. Avkhadiev and A. M. Elizarov

Lipschitz stability of impulsive functional-differential equations

D. D. Bainov and I. M. Stamova $\quad \ldots \ldots \ldots \ldots \ldots \ldots \ldots \ldots \ldots \ldots \ldots \ldots \ldots \ldots$

On monotonicity and superadditivity properties of the entropy function

S. S. Dragomir and C. J. Goh

Optimal control for quasilinear retarded parabolic systems

Liping Pan and Jiongmin Yong

Comparison and linearized oscillation theorems for a nonlinear partial difference equation

B. G. Zhang and Jian-She Yu

Asymptotic behaviour of a nonautonomous cooperative system

Zhu Hongliang and Cui Jingan

On a periodic mutualism model

Yongkun $\mathrm{Li}$

Set by Te $\chi$ Adel, Adelaide, Australia.

Printed by Instant Colour Press, Canberra, Australia. 
The Journal of the Australian Mathematical Society began publication in 1959, and from 1975 appeared in two series, Series A (Pure Mathematics and Statistics) and Series B (Applied Mathematics). Series B is now The ANZIAM Journal and is published in volumes comprising four quarterly parts. There is also a fifth (electronic) part designed for rapid publication (http://jamsb.austms.org.au/). Editor: A. J. ROBERTS, Department of Mathematics \& Computing, University of Southern Queensland, Toowoomba, QLD 4350; aroberts@usq.edu.au. All five parts are refereed. All accepted papers have the option of publication in the electronic part. Information about Series $A$ and other publications of the Society may be found on the inside back cover.

It is the editorial policy of The ANZIAM Journal to accept papers in any field of applied mathematics and related mathematical sciences. Novel applications of mathematics in real situations are especially welcomed. All papers should include some indication of applicability, and an introduction that can be understood by non-specialist readers from the whole applied mathematical community.

Manuscripts (three copies) for publication should be sent to the editor or to an appropriate associate editor at the address given on the inside front cover. Such action is a representation by the author that the manuscript has not been copyrighted or published, and that it is not being considered for publication elsewhere.

Authors are asked to read the section "Preparation of Manuscripts" on the last page.

Irrespective of the number of authors, 50 free offprints of each copy are provided; additional offprints may be ordered at the expense of the authors.

Excessive costs incurred by the Society through corrections to or withdrawal of articles may be charged to the authors concerned.

The ANZIAM Journal (ISSN 0334-2700) is published quarterly for A \$280.50 (Members A\$42.90) per annum by the Australian Mathematical Society, Mathematics Department, Australian National University, ACT 0200, Australia. Send address change notices to Dr A. Howe, Journal of the Australian Mathematical Society, Mathematics Department, Australian National University, ACT 0200, Australia. 